Abstract LL-08 Table 1 Effect Sizes and means (SD) for overall effectiveness of WWE from baseline and follow-up

\begin{tabular}{llll}
\hline Outcome measures & Baseline mean(SD) & Follow-up mean(SD) & Effect size \\
\hline FACIT Fatigue & $24.1(29.4)$ & $19.8(30.4)$ & 0.14 \\
VAS Fatigue & $49.26(71.19)$ & $33.53(71.16)$ & 0.22 \\
VAS Pain & $31.58(54.11)$ & $28.98(53.27)$ & 0.05 \\
VAS Stiffness & $40.25(65.79)$ & $33.33(63.74)$ & 0.11 \\
\hline
\end{tabular}

Conclusions WWE is a feasible, low-cost program that may decrease SLE-related fatigue and stiffness, improve activity and promote long-term lifestyle changes.

Trial registration ClinicalTrials. gov Identifier: NCT02631005.

\section{LL-09 CHILDHOOD ARTHRITIS AND RHEUMATOLOGY RESEARCH ALLIANCE/LUPUS FOUNDATION OF AMERICA CHILDHOOD ONSET SYSTEMIC LUPUS ERYTHEMATOSUS RESEARCH PRIORITIZATION SURVEY}

1,25tacy P Ardoin* ${ }^{3}$ Lyna Merzog, 1,3Aimee Hersh. ${ }^{1}$ Childhood Arthritis and Rheumatology Research Association, USA; ${ }^{2}$ Departments of Pediatrics and Internal Medicine, Ohio State University, USA; ${ }^{3}$ Lupus Foundation of America, USA; ${ }^{4}$ Department of Pediatrics, University of Utah, USA

\subsection{6/lupus-2018-Ism.119}

Background Compared to adult-onset SLE, childhood onset SLE (cSLE) tends to present with more severe disease and more widespread organ involvement. Additionally, cSLE associated with higher mortality and damage accrual. However, despite the severity of the disease few commonly used SLE medications have been rigorously studied in children, and important knowledge gaps remain concerning the epidemiology, natural history, pathophysiology, genetics and optimal treatment of cSLE. In order to identify high priority areas for future research in cSLE, the Lupus Foundation of America (LFA) and the Childhood Arthritis and Rheumatology Research Alliance (CARRA) partnered to perform and cSLE research prioritization survey.

Methods Using information from the 2013 CARRA Lupus Group Research Prioritization Exercise and the most recent LFA Parent and Caregiver Survey, we identified areas of clinical and research need in cSLE. A work group was assembled to develop and refine survey questions which ask clinicians and researchers to rank research topics that are most urgent advance patient outcomes. The survey was programmed using Qualtrics software. The survey was beta tested by a separate group of clinicians and researchers. The proposed survey administration was deemed not to be human subjects research by the University of Utah and Nationwide Children's Institutional Review Boards. Descriptive statistics will be used to analyze survey responses.

Results The survey will be administered to members of CARRA and also to collaborators in the inflammatory skin disease interest group of the Pediatric Dermatology Research Association (PeDRA) and the glomerulonephritis interest group of the Midwestern Pediatric Nephrology Consortium (MWPNC). Together, these organizations include over 800 clinicians and researchers.

Conclusions The results of the CARRA/LFA Research Prioritization Survey will be used to guide funding programs for
cSLE within the Lupus Foundation of America, to share with potential donors, and to use in advocacy efforts promoting state and federal funding for cSLE.

Acknowledgements This study is supported by funding the Lupus Foundation of America and the Childhood Arthritis and Rheumatology Research Alliance.

\section{Tissue Damage}

\section{TD-01 NOVEL IL-16/MIR-125 AXIS CONTROLS NEUTROPHIL RECRUITMENT IN PRISTANE-INDUCED LUNG INFLAMMATION}

${ }^{1} S$ Smith, ${ }^{2} \mathrm{DJ}$ Wallace ${ }^{*},{ }^{1,2} \mathrm{CA}$ Jeffries. ${ }^{1}$ Royal College of Surgeons, Dublin, Ireland; ${ }^{2}$ CedarsSinai Medical Center, Los Angeles, CA

\subsection{6/lupus-2018-Ism. 120}

Background Severe lung inflammation and alveolar hemorrhage can be life threatening in lupus patients. Neutrophil influx is the key driver of this pathology. MiR-125a expression is decreased in patients with lupus pneumonitis. IL-16 is a novel target for mir-125a.

Methods Sera and monocytes from 42 SLE patients, 16 with pulmonary involvement was obtained. Gene expression profiles of monocytes from healthy controls and SLE patients were analyzed by qPCR and correlated with disease activity and organ involvement. Monocytes were transfected with mir-125a mimics and antagomirs and effects on IL-16 mRNA determined by qPCR. Biotin-labelled mir-125a mimics were immunoprecipitated from monocytes and IL-16 confirmed as a direct target by qPCR. Autoimmune lung inflammation was measured following intraperitoneal injection of pristane followed by FACs and qPCR analysis of cell populations in the lung.

Results Reduced miR-125a and enhanced IL-16 expression in SLE patient monocytes was observed and found to correlate with lung involvement. Furthermore, in the pristane model of acute 'SLE-like' lung inflammation, and alveolar hemorrhage, we observe reduced pulmonary miR-125a and enhanced IL-16 expression. Neutrophil infiltration was markedly reduced in the peritoneal lavage of pristine-treated IL-16 deficient mice and elevated following intranasal delivery of IL-16. Moreover, a miR-125a mimic reduces pristane-induced IL-16 expression and neutrophil recruitment, and rescues lung pathology. Mechanistically, IL-16 acts directly on the pulmonary epithelium and markedly enhances neutrophil chemoattractant expression both in vitro and in vivo, whilst the miR-125a mimic can prevent this.

Conclusions A miR-125a mimic reduces pristane-induced IL-16 expression and neutrophil recruitment, and rescues lung pathology. Mechanistically, IL-16 acts directly on the pulmonary epithelium and markedly enhances neutrophil chemoattractant expression both in vitro and in vivo, while the miR-125a mimic can prevent this. A novel role for miR-125a/IL-16 in regulating lung inflammation suggests that this axis may be a therapeutic target for management of acute lung injury in SLE.

Acknowledgement Support provided by NIH Grant (HL0127384), Lupus Research Allliance (ALR/TIL332436), Irish Research Council (GO1PD/2014/512). 